\title{
A Counterexample to the Second Law of Thermodynamics
}

\author{
José C Íñiguez ${ }^{1}$ \\ ${ }^{1}$ Douglas, AZ, USA \\ Correspondence: José C Íñiguez, $122721^{\text {st }}$ Street, Douglas, AZ 85607, USA. Tel: 1-520-805-0010. E-mail: \\ iniguez.jose@gmail.com
}

Received: March 7, 2014 Accepted: April 17, 2014 Online Published: April 29, 2014

doi:10.5539/apr.v6n3p100

URL: http://dx.doi.org/10.5539/apr.v6n3p100

\begin{abstract}
The concatenation of one cycle in the operation of a reversible heat engine with that process in which the engine's work output is irreversibly degraded into heat at the temperature of the cold reservoir, transforms the effects of the former into a couple of irreversible heat transfers. One of these heat transfers is found to be credited, via thermodynamic-sanctioned procedures, with two different entropy changes. A logical analysis centered on the principle of contradiction rejects one of these entropy changes as well as the thermodynamic notion leading to it. The rejected notion, the one assigning a zero entropy change to the heat-to-work transformation, brings to light a counterexample to the second law of thermodynamics in the form of an isothermal and reversible process with a different from zero total entropy change.
\end{abstract}

Keywords: law of increasing entropy, constant entropy reversibility criterion, counterexample, heat to work transformation, entropy, negentropy

\section{Introduction}

No one has expressed the true reach of the law of increasing entropy in simpler terms than Roger Caillois: "Clausius and Darwin cannot both be right" (Prigogine, 1980). Even now, as the following quotes attest, the need is felt for a new theoretical construction capable of doing what Prigogine's Dissipative Structures paradigm was supposed to have accomplished: solving the paradox represented by the second law's future of decay, and nature's incessant success in turning chaos into order. Thus while Farmer states: "I'd like to believe (life) is described by some counterpart of the second law of thermodynamics-some law that would describe the tendency of matter to organize itself, and that would predict the general properties of organization we'd expect to see in the universe" (Waldrop, 1992); Kauffman, on his part, inquires "Could there possibly be a fourth law of thermodynamics for open thermodynamic systems, some law that governs biospheres anywhere in the cosmos or the cosmos itself" (Kauffman, 2004)?

As will here be shown, no fourth law is needed to bring self-organizing phenomena into the realm of thermodynamics, but a simple elimination of a theoretical flaw hidden among the notions sustaining the edifice of what is known as the law of increasing entropy. The construction arising from this correction manages to bring together, in a simple equation, the opposites at play in natural processes: the entropy increasing effects associated to the irreversible degradation of work into heat and the entropy decreasing effects of work creation via the upgrading of heat into work.

\section{Background}

\subsection{Reversible Cyclical Processes}

According to Clausius “.... in a Simple Cyclical Process two variations in respect to heat take place, viz. that a certain quantity of heat is converted into work (or generated out of work), and another quantity of heat passes from a hotter into a colder body (or vice versa)...The relation between these two transformations is...that which is to be expressed by the Second Main Principle" (Clausius, 1879a).

For the case of a reversible heat engine absorbing a cyclical amount heat $Q_{h}$ from a hot reservoir of temperature $T_{h}$, these two transformations can be represented via the following self evident notation: $\left[Q\left(T_{h}\right) \rightarrow W\right]_{r e v},\left[Q_{c}\left(T_{h}\right) \rightarrow Q_{c}\left(T_{c}\right)\right]_{r e v}$. Here $Q$ stands for that portion of $Q_{h}$ that ends up as work $(W)$ in an appropriate mechanical reservoir, and $Q_{c}$ for the remaining portion of $Q_{h}$ which is reversibly transferred to a cold reservoir of temperature $T_{c}$, with $\left.T_{h}\right\rangle T_{c}$. From the previous considerations it is understood that 


$$
Q_{h}=Q+Q_{c}
$$

And also that

$$
Q=W
$$

The amount of work produced by this reversible engine per cycle of operation is quantified by the following equation

$$
W=Q_{h}\left(T_{h}-T_{c}\right) / T_{h}
$$

Here the term $\left(T_{h}-T_{c}\right) / T_{h}$ represents the efficiency of the reversible engine. It quantifies the fraction of every unit of heat coming out of the hot reservoir that ends up transformed into the superior form of energy we call work.

It was in terms of these two transformations that Clausius originally expressed what was to be known as the second law of thermodynamics "...the algebraic sum of all the transformations which occur in a cyclical process must always be positive or in the limit equal to zero..." (Clausius, 1879b). Later on this statement was generalized in the following terms "...The theorem which... was enunciated in reference to circular processes only...has thus assumed a more general form, and may be enunciated thus:- The algebraic sum of all the transformations occurring in any alteration of condition whatever can only be positive, or as an extreme case, equal to nothing..." (Clausius, 1862). For the reversible heat engine being considered and in terms of the previously introduced notation, these statements find the following mathematical representation

$$
\Delta S\left[Q\left(T_{h}\right) \rightarrow W\right]_{r e v}+\Delta S\left[Q_{c}\left(T_{h}\right) \rightarrow Q_{c}\left(T_{c}\right)\right]_{r e v}=0
$$

Equation (4) embodies the zero total entropy principle i.e. the form taken by the second law of thermodynamics when applied to reversible processes.

Serves to note here that at difference with current thermodynamics which describes the total or universe entropy change of a given process in terms of the combined entropy changes of the bodies involved in it, Clausius approach does it in terms of what might be called 'elementary processes' or 'transformations' such as the transformation of heat into work, the transformation of heat between two bodies, the transformation of reactants into products in a chemical reaction, the degradation of heat into work, etc. The entropy change of a given transformation is this way the resultant of the entropy contributions of each and every one of the bodies in it participating. If a body takes part in a given process then its contribution, either individual or as part of a collective, can be identified either as a component of a transformation or as a transformation itself. The summation of the entropy changes of all the transformations subsumed by a given process determines, on its part, its total entropy change.

The identification of the individual entropy changes for the two transformations appearing in Equation (4) can be accomplished via the thermodynamic notion that associates a zero entropy change to the production of work out of heat. Thus, when performing the entropy balance for a reversible heat engine Pitzer and Brewer tells us that in it "...the work term does not appear...since it involves no entropy..." (Pitzer \& Brewer, 1961a). The justification for this position appears to be linked to the fact that the effect of $\left[Q\left(T_{h}\right) \rightarrow W\right]_{\text {rev }}$, as well as that of its opposite $\left[W \rightarrow Q\left(T_{h}\right)\right]_{\text {rev }}$ finds manifestation in the change of condition of a purely-mechanical body (be it the rising or lowering of weights, the elongation/compression of a spring, or any other equivalent mechanism), restricted by its very nature to reversible ergo isentropic processes. This rationale takes the form $\Delta S_{w t}=0$ in the entropy balances performed by Bent (1965a) for a number of processes, and is also found in the following quote by Barrow "...we are at liberty, you should recognize, to ascribe any features to this new entropy function that we like, the requirement being that we construct a function that is self consistent and allows us to form a useful expression for the second law. In this way we further specify that, for all processes, $d S_{\text {mech res }}=0$ " (Barrow, 1973). Here $d S_{\text {mech res }}=0$ refers to the entropy change for the mechanical reservoir. The previous statements can all be found subsumed one way or another in the thermodynamic definition of a mechanical reservoir (or reversible work source) as "A system enclosed by adiabatic impermeable walls in which all processes of interest are quasistatic..." (Tschoegl, 2000). An equivalent definition can be found in Callen (2007).

According to the previous considerations, the entropy change for the heat-to-work transformation taking place in a reversible heat engine can be written as 


$$
\Delta S\left[Q\left(T_{h}\right) \rightarrow W\right]_{r e v}=0
$$

The combination of Equations (4) and (5) leads, in turn, to

$$
\Delta S\left[Q_{c}\left(T_{h}\right) \rightarrow Q_{c}\left(T_{c}\right)\right]_{r e v}=0
$$

Note that these entropy changes conform to the zero total entropy change demanded by the second law for reversible processes. The fact that each of these transformations takes into consideration the entropy contribution of all the bodies in it participating makes each of them a universe in itself (and a sub-universe of the heat engine), and their respective entropy changes total entropy changes.

\subsection{The Irreversible Transfer of Heat}

The entropy change for the direct, irreversible transfer of an amount of heat $Q$ from a hot reservoir of temperature $T_{h}$ to a cold reservoir of temperature $T_{c}$ is known to be (Bevan-Ott \& Boerio-Goates, 2000)

$$
\Delta S\left[Q\left(T_{h}\right) \rightarrow Q\left(T_{c}\right)\right]_{i r r}=-\frac{Q}{T_{h}}+\frac{Q}{T_{c}}
$$

In correspondence with the fact that this irreversible process takes place with the sole involvement of the said reservoirs, we find its entropy change determined by the summation of the entropy changes of these two bodies. Thus, $-Q / T_{h}$ and $Q / T_{c}$ respectively represent the entropy changes of the hot and cold reservoirs due to the release, in the former, and the absorption, in the latter, of the amount of heat $Q$.

The validity of this result, it needs to be pointed out, stems from the validity of the notions on which it stands: the first law of thermodynamics $(d U=\delta Q-\delta W)$ and the definition of the entropy, $d S=\delta Q_{\text {rev }} / T$. The fact that a heat bath or heat reservoir is a constant temperature body capable of exchanging heat but not work $(W=0)$ allows any of its changes to be described by $d U=d Q$. This equation makes evident the fact that any heat exchanged by a heat reservoir is equal to the change of a point function. Being this so "the change of state of the heat bath is thus entirely determined by the heat transfer $d Q$ and $d S$ is therefore the same whether this transfer is reversible or not." (Denbigh, 1968). Consequently, the entropy change of a heat reservoir of temperature $T$ becomes simply $\Delta S=Q / T$. It is this equation the one producing the entropy changes of Equation (7).

It should also be noted from Equation (7) that with the exception of the zero entropy change arising at that limit case of reservoirs of the same temperature, in any other situation in which as assumed $\left.T_{h}\right\rangle T_{c}$, the entropy change will be positive and as such in agreement with the law of increasing entropy, the form adopted by the second law of thermodynamics in the case of irreversible processes such as the transfer of heat here being considered.

The re-expression of the right hand side of Equation (7) as $\left(Q\left(T_{h}-T_{c}\right) / T_{h}\right) / T_{c}$ permits, with the aid of Equation (3), the identification of $\left(Q\left(T_{h}-T_{c}\right) / T_{h}\right)$ with the work $Q$ could have produced had it been fed to a reversible engine working between the said two reservoirs. On this perspective it can be properly called the work lost by the irreversible transit of $Q$. Its representation as $\left[W_{l o s t}(Q)\right]$ permits giving Equation (7) the following form

$$
\Delta S\left[Q\left(T_{h}\right) \rightarrow Q\left(T_{c}\right)\right]_{i r r}=\left[W_{\text {lost }}(Q)\right] / T_{c}
$$

The term $\left[W_{\text {lost }}(Q)\right]$ was chosen to distinguish Equation (8) from that other equation of similar form found in second law thermodynamics which quantifies the entropic effect of the degradation of actual work into heat "If an amount of work $\delta W$ is degraded to heat at temperature $T$, the increase in entropy is $d S_{i r r}=\delta W / T$ " (Pitzer \& Brewer, 1961b).

The work degrading process to which the previous quote makes reference and the entropy change by it produced, for the purpose of the discussions that follow, will be represented as follows

$$
\Delta S\left[W \rightarrow Q\left(T_{c}\right)\right]_{i r r}=W / T_{c}
$$

\subsection{Work Production in Cyclical Processes}

As known, the cyclical evolution of a reversible heat engine is produced by the concatenation of a reversible and isothermal expansion at the temperature of the hot reservoir; an adiabatic and reversible expansion taking the working substance, which will heretofore be taken as an ideal gas, from the temperature of the hot reservoir to that of the cold reservoir; an isothermal and reversible compression at the temperature of the cold reservoir, and finally, closing the cycle, an adiabatic and reversible compression taking the gas back to its initial condition. 
On reason of the fact that in cyclical processes the heat-to-work and work-to-heat transformations take place along the isothermal and reversible processes is that a closer look to these processes will be taken in what follows.

Besides the ideal gas appropriately contained in a cylinder fitted with a frictionless and weightless piston, an isothermal and reversible process demands the concourse of a heat reservoir of the same temperature of the gas, and also of a mechanical reservoir to store/supply the work produced/required along the expansion/compression. In the case of an isothermal and reversible expansion (IRE), the ideal gas manages to quantitatively transform into work all of the heat absorbed from the reservoir. Now, before any amount of heat $\delta Q$ can be transformed into work, it has first to flow from the reservoir to the gas. This allows us to recognize that two concomitant processes are taking place along the expansion: The transfer of heat between two bodies of the same temperature, and the actual transformation of heat into work by the expanding gas. According to the notation above introduced, the total entropy change for this process can be written as follows

$$
\Delta S[I R E]=\Delta S[Q(T) \rightarrow Q(T)]_{\text {rev }}+\Delta S[Q(T) \rightarrow W]_{\text {rev }}=0
$$

That Equation (10) is equal to zero finds explanation in the fact that the entropy changes of the two transformations in it appearing are also zero: that of the heat transfer on reason of it taking place between two bodies of the same temperature; and that of the heat-to-work transformation on reason of Equation (5). This equation is the mathematical representation of the second law of thermodynamics in the form it applies to isothermal and reversible expansions. The fact that $\Delta S[Q(T) \rightarrow W]_{r e v} \neq 0$ is incompatible with this principle will allow the construction of a counterexample to it.

Serves to note that in an (IRE) taken by itself, i.e. not as part of a heat engine, full conversion of heat into work is achieved at the price of leaving the gas in an expanded condition. In order to have a second work-producing round the work produced will have to be expended in full in taking the gas back to its compressed original position, so no work will remain at the end of this peculiar cycle. The continuous transformation of heat into work needs to make use of a heat engine and this, in turn demands a cyclical process such as the one described at the beginning of this section. Any of these engines, even if reversible, and excluding reservoirs of infinite or absolute zero temperatures, will never be able to achieve the full conversion of heat into work.

\subsection{The Principle of Contradiction}

Aristotle's principle of contradiction will be the criterion used in this work to identify which of two contradictory statements is at fault. For the purpose of this task this principle will be expressed as follows: $X$ cannot be both $X$ and not $X$ at the same time and in the same respect. Violation of this principle implies a contradiction. The contradiction consists in that even if being $X$ excludes the possibility of not being $X$ at the same time and in the same respect, both of these positions are taken as true when in reality "they divide the true and the false between them" i.e., "one member of the pair must be true and the other false" (Horn, 2010). Any set of statements involving contradictions becomes, this way, logically inconsistent.

\section{The Contradictions of Second Law Thermodynamics}

\subsection{From One Cycle of a Reversible Heat Engine to a Couple of Irreversible Heat Transfers}

The effects of one cycle in the operation of the reversible heat engine discussed in Section 2.1 have been depicted in Figure 1(a). There, the (implicit) engine is shown transferring the amount of heat $Q_{c}$ from the hot to the cold reservoir, as well as transforming into work $(W)$ the amount of heat $Q$ of temperature $T_{h}$. As before stated, $Q_{h}=Q+Q_{c}$. If this cycle is now concatenated with that process shown in 1 (b) in which $W$ is irreversibly degraded, say via a frictional mechanism, into an equivalent amount of heat ending up in the cold reservoir, we will see the original effects of the reversible engine reduced to the two irreversible heat transfers shown in 1(c). In order to understand the transit from 1(a)+1(b) to 1(c) let us consider the following two facts.

1) That the concatenation of the heat-to-work transformation shown in 1(a), with the work-to-heat transformation shown in 1(b), produces as its sole effect the irreversible transfer of $Q$ from the hot to the cold reservoir shown in 1(c) as $Q_{i r r}$ : Having left the hot reservoir of temperature $T_{h}$ to be transformed into $W$ in $1(\mathrm{a}), Q$ reappears in 1(b) entering the cold reservoir upon the transformation of $W$ back into heat taking place there. Therefore

$$
\left[Q\left(T_{h}\right) \rightarrow W\right]_{\text {rev }}+\left[W \rightarrow Q\left(T_{c}\right)\right]_{i r r}=\left[Q\left(T_{h}\right) \rightarrow Q\left(T_{c}\right)\right]_{i r r}
$$

As should be noted from Figure 1, the other transformation taking place in 1 (a) $\left[Q_{c}\left(T_{h}\right) \rightarrow Q_{c}\left(T_{c}\right)\right]_{\text {rev }}$ reappears in $1(\mathrm{c})$ as $Q_{c, i r r}$, shorthand for $\left[Q_{c}\left(T_{h}\right) \rightarrow Q_{c}\left(T_{c}\right)\right]_{i r r}$.

2) That while the availability of $W$ in $1(\mathrm{a})$ is the reason behind the reversible condition of the two 
transformations there taking place - the simple feeding of $W$ to the inverse cycle has the effect of restoring the initial state without changes being left elsewhere; It is the unavailability of work in 1(c) what explains the irreversible condition of the two heat transfers there depicted. The reason is simple. The transfer back in 1(c) of $Q$ or $Q_{c}$, or both, from the cold to the hot reservoir demands the expenditure of work. The fact that none is here available means that such a reversion can only take place at the price of leaving a permanent change in that body called to supply the required amount of work.

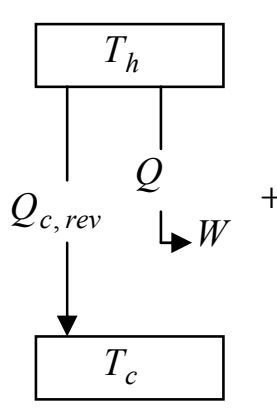

(a)

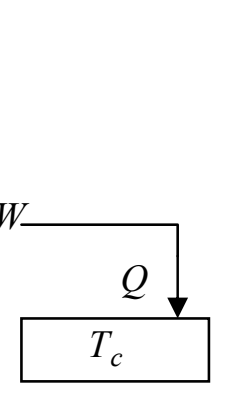

(b)

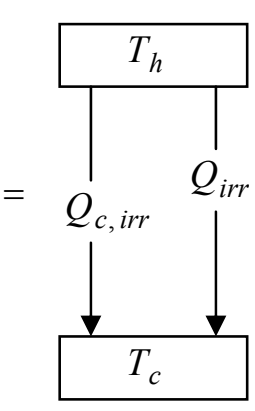

(c)

Figure 1. Out of the amount of heat $Q_{h}$ released by the hot reservoir of temperature $T_{h}$, the reversible heat engine implicit in (a) manages to transfer the portion $Q_{c}$ to the cold reservoir of temperature $T_{c}$, and to transform the difference $Q=Q_{h}-Q_{c}$ into the equivalent amount of work $W$. The further degradation of $W$ into heat at the temperature of the cold reservoir shown in (b) has the effect of reducing the original engine effects to those of the two irreversible heat transfers shown in (c). Here $Q_{c, r e v} Q_{c, i r r}$ and $Q_{\text {irr }}$ stand, respectively, for $\left[Q_{c}\left(T_{h}\right) \rightarrow Q_{c}\left(T_{c}\right)\right]_{r e v},\left[Q_{c}\left(T_{h}\right) \rightarrow Q_{c}\left(T_{c}\right)\right]_{i r r}$, and $\left[Q\left(T_{h}\right) \rightarrow Q\left(T_{c}\right)\right]_{i r r}$

\subsection{The First Entropy Change for $\left[Q\left(T_{h}\right) \rightarrow Q\left(T_{c}\right)\right]_{i r r}$}

The irreversible nature of both of the heat transfers shown in 1(c) makes them identical in nature to that described in Section 2.2. If so, their respective entropy changes can, at the light of that expressed by Equation (7), be written as follows

$$
\begin{gathered}
\Delta S\left[Q\left(T_{h}\right) \rightarrow Q\left(T_{c}\right)\right]_{i r r}=-\frac{Q}{T_{h}}+\frac{Q}{T_{c}} \\
\Delta S\left[Q_{c}\left(T_{h}\right) \rightarrow Q_{c}\left(T_{c}\right]_{i r r}=-\frac{Q_{c}}{T_{h}}+\frac{Q_{c}}{T_{c}}\right.
\end{gathered}
$$

The addition of Equations (12) and (13) leads, with the concourse of Equation (3), the facts that $Q_{h}=Q+Q_{c}$, and $Q=W$, as well as some algebraic rearrangements, to the following equivalent expressions for the total entropy change of process $1(\mathrm{c})$ :

$$
\Delta S[1(c)]_{i r r}=\left(-\frac{Q}{T_{h}}+\frac{Q}{T_{c}}\right)+\left(-\frac{Q_{c}}{T_{h}}+\frac{Q_{c}}{T_{c}}\right)=-\frac{Q_{h}}{T_{h}}+\frac{Q_{h}}{T_{c}}=\frac{Q_{h}\left[\left(T_{h}-T_{c}\right) / T_{h}\right]}{T_{c}}=\frac{Q}{T_{c}}=\frac{W}{T_{c}}
$$

\subsection{The Second Entropy Change for $\left[Q\left(T_{h}\right) \rightarrow Q\left(T_{c}\right)\right]_{i r r}$}

The entropy change for the irreversible transfer of $Q$ shown in 1(c) can, in attention to Equation (11), be written as follows

$$
\Delta S\left[Q\left(T_{h}\right) \rightarrow Q\left(T_{c}\right)\right]_{i r r}=\Delta S\left[Q\left(T_{h}\right) \rightarrow W\right]_{\text {rev }}+\Delta S\left[W \rightarrow Q\left(T_{c}\right)\right]_{i r r}
$$

Further substitution in the previous expression of Equations (5) and (9) produces

$$
\Delta S\left[Q\left(T_{h}\right) \rightarrow Q\left(T_{c}\right)\right]_{i r r}=0+W / T_{c}
$$

The combination of Equation (16) with the appropriate equivalent expressions given by Equation (14) for $W / T_{c}$ leads to

$$
\Delta S\left[Q\left(T_{h}\right) \rightarrow Q\left(T_{c}\right)\right]_{i r r}=0+\left[\left(-\frac{Q}{T_{h}}+\frac{Q}{T_{c}}\right)+\left(-\frac{Q_{c}}{T_{h}}+\frac{Q_{c}}{T_{c}}\right)\right]=0+\left[-\frac{Q_{h}}{T_{h}}+\frac{Q_{h}}{T_{c}}\right]
$$


A simple comparison of Equation (12) with Equation (17) makes evident the fact that in the latter $\left[Q\left(T_{h}\right) \rightarrow Q\left(T_{c}\right)\right]_{i r r}$ is credited with the whole of the entropy change for process 1(c); in other words, here the irreversible transfer of $Q$ appears responsible not only for its own entropy change, but also for that of the irreversible transfer of $Q_{c}$. The fact that Equations (12) and (17) define the entropy change of the very same process in terms of contradicting notions, leads to the conclusion that one of these equations is at fault. The validation of Equation (7) and by extension that of Equation (12) carried on in Section 2.2 in terms of the first law and the definition of the entropy is evidence enough to put in doubt the result given in Equation (17). To prove that as suspected, such a result is incorrect, is the matter of the following argument which starts recognizing that the entropy change for $\left[Q\left(T_{h}\right) \rightarrow Q\left(T_{c}\right)\right]_{i r r}$ appearing in Equation (17) is not given, as should be expected, in terms $Q$, as is done by Equation (12), but in terms of both $Q$ and $Q_{c}$ combined, or equivalently, in terms of $Q_{h}$. In other words, even if the heat transferred is $Q$, the effects correspond to $Q_{h}$. The notion embedded in Equation (17) that $Q$ can be both: $Q$ when transferred from the hot to the cold reservoir and not $Q$ in regard to the entropy change produced by this transfer violates the principle of contradiction. Note that both statements refer to that moment in the process in which the transfer of $Q$ takes place, and are given in respect to the concatenation of processes depicted in Figure 1. Notwithstanding the fact that being $Q$ excludes the possibility of not being $Q$ at the same time in the same respect, Equation (17) takes both of these statements as true. It is on reason of this that the entropy change given by Equation (17) for $\left[Q\left(T_{h}\right) \rightarrow Q\left(T_{c}\right)\right]_{i r r}$ is rejected as false.

It needs to be mentioned here that there exists another perspective, independent from the one just discussed, from which the logical inconsistency of Equation (17) can be approached (Íñiguez, 2011, 2012). It will be briefly mentioned in Section 3.8.

\subsection{The Logical Imbroglio of Second Law Thermodynamics}

The rejection on logical grounds of Equation (17), and by extension of Equation (16) on which it originates, eliminates the contradiction of having two different entropy changes for the same process. This result simply confirms the truthfulness of Equation (12), previously validated in terms of the notions sustaining it. Now, even if Equations (16) and (17) are out of the way, it is imperative to identify the reason of their logical inconsistency. This will be done below.

Let us start this discussion by writing the following expression resulting from the appropriate combination of Equations (12) and (15)

$$
\Delta S\left[Q\left(T_{h}\right) \rightarrow Q\left(T_{c}\right)\right]_{i r r}=\Delta S\left[Q\left(T_{h}\right) \rightarrow W\right]_{r e v}+\Delta S\left[W \rightarrow Q\left(T_{c}\right)\right]_{i r r}=-\frac{Q}{T_{h}}+\frac{Q}{T_{c}}
$$

A term by term comparison between the two rightmost expressions of the previous equation allows us, on reason of the independence of $\left[Q\left(T_{h}\right) \rightarrow W\right]_{\text {rev }}$ and $Q / T_{h}$ on $T_{c}$; as well as of the independence of $\left[W \rightarrow Q\left(T_{c}\right)\right]_{i r r}$ and $Q / T_{c}$ on $T_{h}$, to make the following identifications

$$
\begin{aligned}
& \Delta S\left[Q\left(T_{h}\right) \rightarrow W\right]_{\text {rev }}=-Q / T_{h} \\
& \Delta S\left[W \rightarrow Q\left(T_{c}\right)\right]_{\text {irr }}=Q / T_{c}
\end{aligned}
$$

The substitution of Equation (20) in Equation (15) permits writing the following expression

$$
\Delta S\left[Q\left(T_{h}\right) \rightarrow Q\left(T_{c}\right)\right]_{i r r}=\Delta S\left[Q\left(T_{h}\right) \rightarrow W\right]_{r e v}+\frac{Q}{T_{c}}
$$

Let us now agree that any claim of correctness for Equation (19) is in opposition not only to the accepted thermodynamic notion embodied by Equation (5) giving a zero entropy change to $\left[Q\left(T_{h}\right) \rightarrow W\right]_{\text {rev }}$ but, as already mentioned in regard to Equation (10), also to the second law in its zero total entropy version. Note now that if in acceptance of Equation (5) we replace $\Delta S\left[Q\left(T_{h}\right) \rightarrow W\right]_{\text {rev }}$ in Equation (21) with a zero, we will be regenerating Equation (16) and through it Equation (17); both of them incorrect on logical grounds. Note then that it is not the $W / T_{c}$ term (or equivalently, $Q / T_{c}$ ) of Equation (16) what makes these equations incorrect. This term, written as $Q / T_{c}$, belongs in the correct equation for $\left[Q\left(T_{h}\right) \rightarrow Q\left(T_{c}\right)\right]_{i r r}$ as attested by Equation (18). It is the zero accompanying $W / T_{c}$ in Equation (16) what allows this term to morph its identity into that of the terms shown in the right hand side of Equation (17). The fact that this zero comes from Equation (5) allows us first of all to identify this equation as the origin of the logical conundrum previously unveiled and as such the true object of logical rejection by the argument of the previous section; and also to confirm the validity of Equation (12) and through it that of Equations (19) and (20).

These results provide us, via the substitution of Equation (19) in Equation (10), with a logically consistent 
counterexample to the second law in the form of a reversible and isothermal ideal gas expansion with a non-zero, actually negative total entropy change of magnitude $-Q / T$, i.e.

$$
\Delta S[I R E]=\Delta S[Q(T) \rightarrow Q(T)]_{\text {rev }}+\Delta S[Q(T) \rightarrow W]_{\text {rev }}=-Q / T
$$

Note that the negative total entropy for the isothermal and reversible expansion makes this process negentropic. Serves well in this regard to recall here that the entropy change given by Equation (19) can also found in Clausius work on the second law (Clausius, 1879c).

\subsection{An Isothermal and Reversible Compression}

The fact that the coupling of a reversible process with its precise inverse brings the universe back to its original condition means that each and every effect of the forward process is reverted by its inverse, and if this is so, the entropy change of the latter must be of equal magnitude but opposite sign to that of the former so that their combination, as corresponds to a point function, produce a net change of zero for this cycle. When applied to the inverse of the (IRE) introduced in Section 2.3 and whose correct entropy change appears quoted in Equation (22) i.e. to an isothermal and reversible compression (IRC), we will have that the following is true

$$
\begin{gathered}
\Delta S[I R C]=\Delta S[Q(T) \rightarrow Q(T)]_{r e v}+\Delta S[W \rightarrow Q(T)]_{r e v}=Q / T \\
\Delta S[W \rightarrow Q(T)]_{r e v}=Q / T
\end{gathered}
$$

The different from zero total entropy changes of the isothermal and reversible ideal gas processes described by Equations (22) and (23) provides a glimpse at the paradigmatic changes subsumed by the replacement of Equation (5) by Equation (19). In order to keep expanding this new view is that the following discussions are presented

\subsection{A Partially Reversible Isothermal Expansion}

Let us consider the isothermal and irreversible expansion depicted in Figure 2. In it a portion $Q_{c}$ out of the amount of heat $Q_{h}$ released by the hot reservoir of temperature $T_{h}$ finds a direct, irreversible path to the cold reservoir of temperature $T_{c}$; with the other portion, $Q$, being transformed into an equivalent amount of work, $Q=W$. Note that the work producing process is reversible on reason of the fact that $Q$ can be returned to the hot reservoir, without any additional change taking place, via an isothermal and reversible compression propelled with $W$. The fact that no work was generated in the transfer of $Q_{c}$ allows us to realize that its transfer back to the hot reservoir cannot be accomplished without leaving a change in that body called to supply the missing work. These considerations permit writing the following equation for the total entropy change of this process

$$
\Delta S[\text { process } 2]=\Delta S_{i r r}+\Delta S_{\text {rev }}
$$

The reversible and irreversible components of the universe entropy change can, on reason of Equation (7) and Equation (19) be written, respectively, as follows

$$
\begin{gathered}
\Delta S_{\text {irr }}=Q_{c}\left(T_{h}-T_{c}\right) /\left(T_{h} T_{c}\right) \\
\Delta S_{\text {rev }}=-Q / T_{h}=-W / T_{h}
\end{gathered}
$$

In terms of which Equation (25) becomes

$$
\Delta S[\text { process } 2]=\left[Q_{c}\left(T_{h}-T_{c}\right) /\left(T_{h} T_{c}\right)\right]-\left(Q / T_{h}\right)
$$




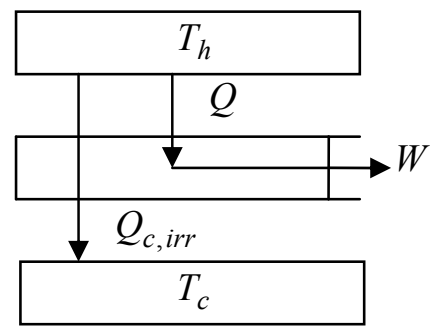

Figure 2. The isothermal expansion depicted is irreversible on reason of the fact that only the portion $Q$ out of the total amount of heat $Q_{h}, Q_{h}=Q+Q_{c}$, released by the hot reservoir of temperature $T_{h}$ is transformed into work. The remaining portion $Q_{c}$ bypasses the work production process by flowing directly to the cold reservoir of temperature $T_{c}$. The irr sub index has been added to $Q_{c}$ to emphasize the irreversible nature of this heat transfer

Note now that this equation correctly reproduces the entropy changes for the two extremes there involved. If $Q_{c}=0$ then no irreversible leaking of heat takes place and $Q=Q_{h}$. In this situation all of the heat released by hot reservoir ends up transformed into work and the total entropy change becomes

$$
\Delta S[\text { process } 2]=-Q_{h} / T_{h}
$$

If, on the other hand, all of the heat released by the hot reservoir finds its irreversible way to the cold reservoir then $Q_{c}=Q_{h}$. In this situation $Q=0$ and no work is at all produced. Therefore

$$
\Delta S[\text { process } 2]=Q_{h}\left(T_{h}-T_{c}\right) /\left(T_{h} T_{c}\right)
$$

It is important realize that work is a common link to the opposite effects shown in Equation (28). Thus while the negentropic contribution of the reversible term given by Equation (27) appears in terms of the work ( $W$ ) output of the process i.e. in terms of the work "gained":

$$
\Delta S_{\text {rev }}=-W_{\text {gained }} / T_{h}
$$

The entropic contribution of the irreversible term given in Equation (26), as previously discussed in reference to Equation (8), can be written in terms of the wasted work producing potential that $Q_{c}$ carries with it in its irreversible transit to the cold reservoir, i.e. in terms of the lost work carried on by $Q_{c}$

$$
\Delta S_{\text {irr }}=\left[W_{\text {lost }}\left(Q_{c}\right)\right] / T_{c}
$$

These last two equations allows us to write the following re-expression of Equation (25)

$$
\Delta S[\text { process } 2]=\left[W_{\text {lost }}\left(Q_{c}\right) / T_{c}\right]-\left(W_{\text {gained }} / T_{h}\right)
$$

This equation, common to other (non-reversible) thermodynamic processes involving the transformation of heat into work (Íñiguez, 2012), allows us to see that in this new perspective the entropy increase associated to the lost work is opposed by the negentropy associated to the work gained. The predominance of one of these opposite over the other depends on the efficiency of the operation. If the efficiency for this isothermal expansion is defined as $\eta=Q / Q_{h}$ then its value at the irreversible extreme $(Q=W=0)$ is zero, while at the reversible end ( $Q=W=Q_{h}$ ) is one. It should be noted that in the zero-efficient irreversible realm in which no work is produced (no work produced, no negentropy) Equation (33) will lose its rightmost term to become, as previously noted in reference to Equation (7), the following re-expression of the law of increasing entropy: $\Delta S$ [process 2$]=\left[W_{\text {lost }}\left(Q_{c}\right) / T_{c}\right]$. The previous argument allows us to see that it is the zero-efficient irreversible limit where the rule of the law of increasing entropy is absolute. In this perspective the second law as we know it correctly describes the domain of irreversible processes. As soon as heat starts finding its way into the superior form of energy we call work, the description of the situation demands the use of Equation (33) or its equivalent re-expressions. It comes apparent from Equation (33) that on reason of being $T_{h}>T_{c}$, every unit of work wasted produces a larger amount of entropy than the negentropy brought forward by every unit of work gained. In this perspective the 'punishment' for wasting work exceeds the 'reward' for gaining it.

Inherent to the previous discussion is the fact that the total entropy of the irreversible isothermal expansion being considered transits from a positive value at $\eta=0$ to a negative value at $\eta=1$. But if this is so, there must be 
an efficiency $\eta_{o}$ at which the total entropy, as a reflection of identical magnitude but opposite sign contributions from the entropic and negentropic processes there taking place, becomes zero. This efficiency can be identified for the process under study by setting Equation (28) equal to zero and solving for $\eta_{o}$. When this done we get

$$
\eta_{o}=\left(T_{h}-T_{c}\right) / T_{h}
$$

Any of these processes taking place with $\eta\left\langle\eta_{o}\right.$ will be entropic. All those for which $\left.\eta\right\rangle \eta_{o}$ will be negentropic, and those complying with $\eta=\eta_{o}$, isentropic.

The previous discussion unveils the fact that the entropy is more than a state function; It is also the indicator of the transition point from entropic to negentropic universes (or vice versa). The separation of the total entropy change of thermodynamic processes in entropic and negentropic contributions allows us to give precise meaning to the notions of thermodynamic chaos and thermodynamic order. The latter finding expression in the negentropic region where the effect of the ordered energy we call work overcomes that of the disordered energy we call heat. In the opposite case it is the former -thermodynamic chaos- the one finding expression. On this perspective the transition from entropic to negentropic universes can be likened with the transition from chaos to order and vice versa.

If we are capable of developing expressions for the work gained appearing in self-organizing phenomena as spatial-functional order i.e. for the work involved in the construction and sustaining of the structures evident in those processes v. g. The rolls or cells appearing in Bénard Convection (Íñiguez, 2010), as well as for the work lost in those processes, we will be able to test via Equation (33) if as posited here, these structures materialize the transitions of these systems from entropic to negentropic, as well as to determine and compare with experiment the transition point at which disorder becomes order.

\subsection{Heat as Mechanical Energy}

Two elements of judgment are required for the argument to be presented below. The first one makes use of the contrast made by Smith and Van Ness between heat and work, as follows "One of the clearest interpretations of the second-law principle may be achieved by considering the differences between the two forms of energy, heat and work...". In this regard "...experience teaches that there is a difference in quality between heat and work ...". The experimental facts exhibited by these authors in support of this notion are, on the one hand, the practically unrestricted convertibility from one form of work to another, and in the other, the severely restricted convertibility of heat into any form of work. The efficiencies for the latter kind of processes are so low in comparison with those obtained for the transformation of work from one form to another that "...there can be no escape from the conclusion that there is an intrinsic difference between heat and work". This difference may be summarized by saying that "...heat is a less versatile or more degraded form of energy than work" and that "... work might be termed energy of a higher quality than heat" (Smith \& Van Ness, 1965). Note that the previous considerations imply a common quality for the different forms of mechanical energy, or equivalently, for the different forms of work. These notions find complement in the common interpretation of the total entropy increase in irreversible processes as a "...quantitative measure, or index, of the degradation of energy as work to energy as heat..." (Weber \& Meissner, 1957).

The second element refers to the fact that any process taking place in a conservative mechanical system, such as the inter-conversions of one form of mechanical energy to another, takes place at constant entropy.

Let us now transcribe here the total entropy expression for an isothermal and reversible expansion as written by Bent in accord with current thermodynamic wisdom (Bent, 1965b).

$$
\Delta S[I R E]=-(Q / T)+(Q / T)+\Delta S_{W t .}=0
$$

In the previous equation $\Delta S_{W t}$. refers to the entropy change of the conservative weight-in pulley system used by Bent as mechanical reservoir. In it the weight's position changes in response to the heat-to-work transformation represented as $[Q(T) \rightarrow W]_{\text {rev }}$. Bent's original expression makes use of the internal energy change of the heat reservoir for the quantification of its own entropy change as well as that of the gas. The fact that for a heat reservoir, as previously noted, $\Delta E=Q$, allows re-expressing these entropy changes in terms of $Q$ as shown in Equation (35).

From a comparison between Equations (35) and (10) two things become evident: that the term $\Delta S[Q(T) \rightarrow Q(T)]_{\text {rev }}$ of the latter correctly corresponds with the first two terms of the former, and that for second law thermodynamics, as we know it, the entropy change associated to the weight's displacement $\left(\Delta S_{W t .}\right)$ is identical with that of the thermodynamic process upgrading heat into work, i.e. 


$$
\Delta S_{W t .}=\Delta S[Q(T) \rightarrow W]_{\text {rev }}=0
$$

For the purposes of the coming discussion the two rightmost terms of the previous equation will be written as follows

$$
\Delta S\left[Q(T) \rightarrow E_{W t .}\right]_{\text {rev }}=0
$$

In it the work term has been substituted by its equivalent, the energy of the weight.

Let us now consider the situation in which the lifting of the weight in Bent's mechanical reservoir is produced via the uncoiling of a nonfrictional spring previously coupled to it. The fact that this process involves no other change but the transformation of the elastic energy of the spring into the gravitational-potential energy of the weight makes it, as previously noted, an isentropic process. These considerations are reflected in the following expression

$$
\Delta S\left[E_{\text {spring }} \rightarrow E_{W t}\right]_{\text {rev }}=0
$$

As made evident by a comparison between Equations (37) and (38), for current thermodynamics the transformation of heat into mechanical energy in Equation (37) is entropically indistinguishable from the transformation of elastic energy into gravitational-potential energy represented in Equation (38); in other words, heat as an energy form is, in regard to Equation (37), indistinguishable from any other form of mechanical energy, as it is capable of transiting, of morphing into the gravitational-potential energy of the weight at constant entropy. This conclusion is, however, in opposition to experience. As previously noted, heat is intrinsically different from mechanical energy. In negating this experience supported fact, the second law negates itself, as its foundation rests precisely in the notion that these two energy forms are intrinsically different.

It needs to be stressed that what has been faulted is not the isentropic nature of the mechanical reservoir, but its identification - shown in Equation (36) - with the thermodynamic process responsible for the heat-to-work transformation.

The transformation of heat into work and the entropy change to it associated is not, as current thermodynamics posits, an attribute of the mechanical reservoir but of the collective of bodies in it involved. In the case of the (IRE) it is an attribute of the heat reservoir, the gas, and the mechanical reservoir. Take any of these bodies out and the heat-to-work transformation cannot take place. The extreme reductionist position of current thermodynamics in terms of the entropies of 'bodies', finds its limit in the impossibility to assign the entropy change of the heat-to-work-transformation to any of the bodies there involved.

When Bent's total entropy change for the isothermal and reversible ideal gas expansion given in Equation (35) is looked upon at the light of the fact that transformation $[Q(T) \rightarrow W]_{\text {rev }}$ involves all the bodies taking part in it -mechanical reservoir included- it becomes evident that it needs to be corrected via the replacement of $\Delta S_{W t}$. by $\Delta S[Q(T) \rightarrow W]_{\text {rev }}$. This simple replacement embodies the conceptual shift separating current second law thermodynamics from the formulation here being introduced, i.e.

$$
-(Q / T)+(Q / T)+\Delta S_{W t} \rightarrow-(Q / T)+(Q / T)+\Delta S[Q(T) \rightarrow W]_{r e v}
$$

Materializing in the identification of $\Delta S[Q(T) \rightarrow W]_{\text {rev }}$ with $-Q / T$ this change produces the correct result for the total entropy change of an isothermal and reversible ideal gas expansion:

$$
\Delta S[I R E]=-Q / T
$$

\subsection{The Spontaneous Flow of Heat From a Colder to a Hotter Body}

A different perspective to the contradictions incurred by second law thermodynamics, as we know it, in sanctioning two contradictory entropy changes for $\left[Q\left(T_{h}\right) \rightarrow Q\left(T_{c}\right)\right]_{i r r}$, can be unveiled through the following argument.

Let us then assume that that the following equation, a combination of Equations (14) and (17), is true

$$
\Delta S[1(c)]_{i r r}=\Delta S\left[Q\left(T_{h}\right) \rightarrow Q\left(T_{c}\right)\right]_{\text {irr }}
$$

The problem with this equations starts once we recognize the fact, evident from Figure 1(c) that $\Delta S[1(c)]_{i r r}$ is also given by the following equation

$$
\Delta S[1(c)]_{i r r}=\Delta S\left[Q\left(T_{h}\right) \rightarrow Q\left(T_{c}\right)\right]_{i r r}+\Delta S\left[Q_{c}\left(T_{h}\right) \rightarrow Q_{c}\left(T_{c}\right)\right]_{i r r}
$$

A comparison between the last two equations highlights the point that the validity of current second law 
thermodynamics position embodied in Equation (41) presupposes the validity of the following relation

$$
\Delta S\left[Q_{c}\left(T_{h}\right) \rightarrow Q_{c}\left(T_{c}\right)\right]_{i r r}=0
$$

It is through a simple analysis of the previous equation that two important flaws subsumed by this position come to light.

1) The fact that the process to which Equation (43) makes reference is reversible on reason of its entropy change and irreversible on reason of the fact that in 1 (c) it is impossible to transfer $Q_{c}$ back to the hot reservoir without changes remaining elsewhere, attest to the non-equivalence between the constant total entropy reversibility criterion and the possibility of restoring the precise initial condition of the universe. Here we have a reversible process that can't be reverted.

2) The only possibility open for the zero total entropy reversibility criterion to remain valid in regard to what Equation (43) expresses, i.e. the only possibility open for us to trade "irr" for "rev" in Equation (43) demands from $Q_{c}$ the ability to flow of itself, unassisted, from the cold to the hot reservoir. In other words, the validity of the zero total entropy reversibility criterion, and by extension that of the total entropy increasing quality of irreversible processes, demands the non-validity of that principle to which the second law is supposedly equivalent, namely, that "heat cannot, of itself, pass from a colder to a hotter body" (Clausius, 1879d).

\subsection{The Reversible to Irreversible Transition of $\left[Q_{c}\left(T_{h}\right) \rightarrow Q_{c}\left(T_{c}\right)\right]$}

With the problem regarding the entropy change for $\left[Q\left(T_{h}\right) \rightarrow Q\left(T_{c}\right)\right]_{i r r}$ finally settled, there is still the matter of the change experienced by $\left[Q_{c}\left(T_{h}\right) \rightarrow Q_{c}\left(T_{c}\right)\right]$ from $1(\mathrm{a})$ to $1(\mathrm{c})$ consisting in the transition from a reversible condition and a zero entropy change to an irreversible condition and an entropy change of magnitude $-\left(Q_{c} / T_{h}\right)+\left(Q_{c} / T_{c}\right)$.

Let us agree that in 1(a) no lost work is produced by the reversible transit of $Q_{c}$ to the cold reservoir. If this were not so then, contrary to experience, processes capable of outputting larger amounts of work than the reversible work would be possible. The previous consideration explains, via the notion subsumed by Equation (8), the zero entropy change associated to the reversible transfer of $Q_{c}$ (no lost work, no entropy production). This situation changes radically, however, once process 1(b) takes effect. Here, subsumed in the two irreversible transfers depicted in 1(c) we find $W$ reappearing as the dissipated work producing potential (the lost work) carried by these two heat transfers in their irreversible transit to the cold reservoir. The fact that while in 1(a) the transfer of $Q_{c, r e v}$ takes place without lost work and consequently at constant entropy, and that in 1(c) the transfer of $Q_{c, i r r}$ is accompanied with a wasted work producing potential in the amount of $\left(Q_{c}\left(T_{h}-T_{c}\right) / T_{h}\right)$ and an entropy change of $\left(Q_{c}\left(T_{h}-T_{c}\right) / T_{h}\right) / T_{c}$ means that the entropy change for the transit from the former to the latter taking place on reason of $1(\mathrm{~b})$ can be written as

$$
\Delta S\left[Q_{c, i r r}\right]-\Delta S\left[Q_{c, r e v}\right]=Q_{c}\left(T_{h}-T_{c}\right) / T_{h} T_{c}
$$

If so, then in agreement with our previous result shown in Equation (13) we get

\section{Conclusions}

$$
\Delta S\left[Q_{c, \text { irr }}\right]=\Delta S\left[Q_{c, \text { rev }}\right]+\left(Q_{c}\left(T_{h}-T_{c}\right) / T_{h} T_{c}\right)=Q_{c}\left(T_{h}-T_{c}\right) / T_{h} T_{c}
$$

Hailed as the supreme law of nature by Eddington (Nikulov \& Sheehan, 2004), the law of increasing entropy has been here shown to be a logically inconsistent construction. Its correction materializes in an equation taking into account the opposing tendencies at the core of natural phenomena, order and chaos. Even if the evidence is clear and appears unassailable, the final verdict belongs, as Planck has said in this context, to experience "... no more effective weapon can be used by both champions and opponents of the second law than indefatigable endeavor to follow the real purport of this law to the utmost consequences, taking the latter one by one to the highest court of appeal -experience. Whatever the decision may be, lasting gain will accrue to us from such a proceeding, since thereby we serve the chief end of natural science - the enlargement of our stock of knowledge" (Planck, 1990).

\section{References}

Barrow, G. M. (1973). Physical Chemistry (3rd ed., p. 168). New York, NY: McGraw-Hill Book Company.

Bent, H. A. (1965a). The Second Law (pp. 55-57, 77-81). New York, NY: Oxford University press.

Bent, H. A. (1965b). The Second Law (pp. 78-79). New York, NY: Oxford University press.

Bevan-Ott, J., \& Boerio-Goates, J. (2000). Chemical Thermodynamics, Principles and Applications (pp. 80-81). London, UK: Academic Press.

Callen, H. B. (2007). Thermodynamics and an Introduction to Thermostatics (2nd ed., p. 103). New York, NY: 
John Wiley and Sons.

Clausius, R. (1862). On the Application of the Theorem of Equivalence of Transformations to the Internal Work of a Mass of Matter. Philosophical Magazine Series 4, 24(159), 211. http://dx.doi.org/10.1080/14786446208643322

Clausius, R. (1879a). The Mechanical Theory of Heat (pp. 91-92). London, UK: Macmillan and Co. Retrieved from https://archive.org/details/cu31924101120883

Clausius, R. (1879b). The Mechanical Theory of Heat (p. 213). London, UK: Macmillan and Co. Retrieved from https://archive.org/details/cu31924101120883

Clausius, R. (1879c). The Mechanical Theory of Heat (pp. 100-101). London, UK: Macmillan and Co. Retrieved from https://archive.org/details/cu31924101120883

Clausius, R. (1879d). The Mechanical Theory of Heat (p. 78). London, UK: Macmillan and Co. Retrieved from https://archive.org/details/cu31924101120883

Denbigh, K. (1968). The principles of Chemical Equilibrium (2nd ed., p. 40). London, UK: Cambridge University Press.

Horn, L. R. (2010). Contradiction. Stanford Encyclopedia of Philosophy. Retrieved from http://plato.stanford.edu/entries/contradiction

Íñiguez, J. C. (2010). An unusually efficient coupling of Carnot engines. Apeiron, 17(2), 150-172. Retrieved from http://redshift.vif.com/JournalFiles/V17NO2PDF/V17N2INI.pdf

Íñiguez, J. C. (2011). A Thermodynamic Impasse: A constant entropy irreversible process. Physical Chemistry: An Indian Journal, 6(3), 125-130. Retrieved from https://www.researchgate.net/profile/Jose_Iniguez2/

Íñiguez, J. C. (2012). A Critical Study of the Law of Increasing Entropy. Conshohocken, PA: Infinity Publishing Co.

Kauffman, S. (2004). Investigations (p. 2). New York, NY: Oxford University Press.

Nikulov, A., \& Sheehan, D. (2004). The Second Law Mystique. Entropy, 6, 1-10.

Pitzer, K. S., \& Brewer, L. (1961a). Thermodynamics (2nd ed., p. 95). (Revision of Lewis and Randall). New York, NY: McGraw-Hill International Student Edition.

Pitzer, K. S., \& Brewer, L. (1961b). Thermodynamics (2nd ed., pp. 85-86). (Revision of Lewis and Randall). New York, NY: McGraw-Hill International Student Edition.

Planck, M. (1990). Treatise on Thermodynamics (p. 107). New York, NY: Dover.

Prigogine, I. (1980). From Being to Becoming: Time and complexity in the physical sciences (p. 84). New York, NY: W. H. Freeman and Company.

Smith, J. M., \& Van Ness, H. C. (1965). Introduction to Chemical Engineering Thermodynamics (pp. 175-176). Mexico, DF: McGraw-Hill Book Co., International Student Edition.

Tschoegl, N. W. (2000). Fundamentals of Equilibrium and Steady-State Thermodynamics (pp. 36-37). Amsterdam, Holland: Elsevier. http://dx.doi.org/10.1016/B978-044450426-5/50006-9

Waldrop, M. M. (1992). Complexity: The emerging science at the edge of order and chaos (p. 288). New York, NY: Touchstone.

Weber, H. C., \& Meissner, H. P. (1957). Thermodynamics for Chemical Engineers (p.168). New York, NY: John Wiley and Sons.

\section{Copyrights}

Copyright for this article is retained by the author(s), with first publication rights granted to the journal.

This is an open-access article distributed under the terms and conditions of the Creative Commons Attribution license (http://creativecommons.org/licenses/by/3.0/). 